\title{
Proximal Junction Kyphosis in Adult Scoliosis. Best Post-Operative Radiological Predictors. Retrospective Cohort Study
}

\section{Zhang Qi Hong}

Xiangya Hospital Central South University

Daudi Romani Manini ( $\nabla$ daudiromani@yahoo.co.uk)

Central South University Xiangya School of Medicine

\section{Xiang YU Wang}

Xiangya Hospital Central South University

Xuan YU Du

Xiangya Hospital Central South University

Research article

Keywords: proximal junctional kyphosis, adult scoliosis, global sagittal alignment.

Posted Date: October 14th, 2020

DOI: https://doi.org/10.21203/rs.3.rs-89186/v1

License: (9) This work is licensed under a Creative Commons Attribution 4.0 International License. Read Full License 


\section{Abstract}

Background. Proximal junction kyphosis (PJK) is the post-surgical radiographic event seen in the surgical plane after the fusion of spinal deformity. Unfavorable health outcomes have been reported in symptomatic PJK patients. The purpose of this study was to assess which immediate post-surgical radiographic variable from the four proposed formulae is the best in forecasting the future occurrences of PJK in adult scoliosis after undergoing deformity correction.

Methods: Data for adult scoliosis patients who underwent curve correction were extracted from the hospital database. Pelvic and spinal parameters were measured and calculated to compare four predictive formulae for occurrences of PJK. Formula 1区Restoration of hypothetical values of lumbar lordosis (LL) and thoracic kyphosis (TK) according to pelvic incidence (PI). Formula 2: evaluation of global sagittal alignment (GSA). Formula 3: Restoration of the apex of LL to its hypothetical position according to the spine shape. Formula 4: evaluation of positive-sum and negative-sum of ( $L L+T K)$.

Results: The total number of cases was 52 . There were 14 cases of PJK, the incidence of PJK was $26.9 \%$ and the mean age for PJK cases was 63.2 \pm 5.2 . The excellent predictor for occurrences of PJK was formula 3, postsurgical sagittal apexes of lumbar lordosis were located in their hypothetical position in 24 cases, and $12.5 \%$ of these cases developed PJK. While sagittal apexes were not located in hypothetical position in 28 patients, $39.3 \%$ of patients developed PJK. P $=0.03$, OR 4.53, (95\% Cl 1.0918.9). The second good predictor for occurrences of PJK was formula 2. GSA $>45^{0}$ versus $G S A<45^{0} O R=$ 2.5, (95\% Cl, 0.67-9.38), $\mathrm{P}=0.17$. The other two formulae (1 and 4) were not good predictors for occurrences of PJK.

Conclusion: Among the proposed formulae for predicting occurrences of PJK, the position of the sagittal apex of lumbar lordosis is an excellent predictor of the development of PJK followed by GSA. Hypothetical values of $L L$ and TK, positive or negative-sum of $(L L+T K)$ are weak predictors for occurrences of PJK.

Trial registration: "retrospective registered"

\section{Background}

Proximal junction kyphosis (PJK) is the post-surgical radiographic event seen in the surgical plane after the fusion of spinal deformity. In wide view PJK is regarded as a form of adjacent segment disease associated with spinal fusion, mostly occurring after scoliosis or kyphosis surgery $(1,2)$. PJK frequently arises just above the uppermost instrumented vertebrae (UIV). The most accepted PJK definition is the one defined by Glattes et al:(3). Proximal junctional angle (PJA) angle is the angle subtended by the lower endplate of the UIV to the upper endplate of two vertebrae proximal. PJK is said to be present if the sagittal Cobb angle is $\geq 10^{\circ}$ and at least $10^{\circ}$ larger than preoperative measurement(4). The incidence of PJK as reported from the literature varies greatly and ranges from $5-46 \%$ to spinal patients who undergo deformity curve correction. PJK can be diagnosed in the radiographic film as soon as 8 weeks 
postoperatively $(5,6)$. Several risk factors for occurrences of PJK have been reported in the literature including Age more than 55 years, large abnormal preoperative sagittal parameters (high lumbar lordosis and long thoracic kyphosis), application of pedicle screws, thoracoplasty procedures, big curve correction, posterior and combined anteroposterior spinal fusion (i.e., distraction of the posterior tension band and other posterior intervertebral elements), fusion involving the lower lumbar spine and sacrum, osteoporosis and high body mass index $(2,7,8)$. More unfavorable scoliosis research society (SRS) and Oswestry disability index (ODI) scores have been reported in symptomatic PJK patients relative to none-PJK patients. Moreover, it may lead to revision surgery $(1,9)$.

There is no common agreement on direct postoperative radiographic prognostic criteria for PJK that justify close monitoring for the patients. Vialle et al(10) suggested that physiological spinal sagittal balance should serve as a baseline in the assessment of pathological state. They further advocated the restoration of lumbar lordosis (LL) and thoracic kyphosis (TK) to their normal theoretical value according to pelvic incidence (PI). Again in 2012 Yagi et al(2) found that many preoperative patients with imperfect global sagittal alignment (GSA) $\left(T K+\mathrm{LL}+\mathrm{PI}>45^{\circ}\right)$ have a high tendency of developing PJK. They further proposed that high postoperative GSA result in the positive sagittal vertical axis (SVA) and increase the stress for the proximal and distal junctions of instrumentation that lead to the development of PJK. Roussouly et al(11) suggested that the sagittal apex of LL be placed at $L 4$ when $\mathrm{PI}$ is small (in type 1 and type2 lordosis curve). On the other hand, the apex is placed at L3 or L3-L4 disc when PI is larger (in type 3 and type 4 lordosis curve) during deformity correction. They further argued that restoration of sagittal LL apex to its hypothetical standard could radiologically predict the occurrence of PJK and implant breakage. Also, Mendoza et al(12) in a retrospective study of adult spine deformity patients, who underwent curve correction found that PJK occurred in patients whose TK remained bigger compared to LL postoperatively. They further hypothesized that the positive sum of $L L$ and TK was associated with a high rate occurrence of PJK.

This study aimed to assess which immediate post-surgical radiographic variable among the proposed above is the best in forecasting the future occurrences of PJK in adult scoliosis after deformity correction.

\section{Methods}

A retrospective study of 52 adult scoliosis patients who underwent scoliosis curve correction in Xiangya Hospital of Central South University from January 2012 to December 2016 was done. After approval from the institutional review board of Xiangya hospital of Central South University, an informed consent form was obtained from each participant. And all methods were carried out according to the guideline and regulation of the Helsinki declaration(13). Data were retrieved from the hospital database.

Inclusion criteria: (1) Patient with scoliosis deformity (both degenerative scoliosis and adult idiopathic scoliosis) Cobb $\geq 25^{0}$ on the anteroposterior (AP) view. (2) patients aged $\geq 25$ years. (3) patients with a follow-up period of at least 2 years. (4) Patients with all radiological films (preoperative, immediate postoperative, and last follow-up films) available. (5) Patients with lower instrumented vertebra end at L4 
or below. Exclusion criteria: (1) Patients with surgery of other spinal abnormalities such as tumors, tuberculosis, or accident. (2) Patients with incomplete radiographic data. (3) Patients with less than 6 months of follow-up.

\section{Radiographic measurements:}

Both pelvic and sagittal parameters were measured by the method previously described by Lafage et al(14) and Lagaye et al(15). This was done independently by two authors and the differences between them were solved through discussion with the $3^{\text {rd }}$ author. TK angle was measured from the upper endplate of T4 and the lower endplate of T12. LL angle was measured from the upper endplate of L1 to the upper endplate of S1. PI angle was measured between the line drawn perpendicular to the sacral endplate at its center and the line drawn from the center of the sacral endplate to the midpoint of the bicoxofemoral axis. PT angle was measured between the line joining the center of the sacral endplate to the center of the bicoxofemoral axis and the vertical. Sacral slope (SS) was the angle formed between the horizontal and superior sacral endplate of S1. PJA was measured from the lower endplate of the UIV to the upper endplate of 2 levels superior to the UIV. Table 1 shows both preoperative and postoperative spinal pelvic parameters.

Immediate postoperatively 4 formulae were used to forecast the future occurrence of PJK as follows:

Formula 1: Hypothetical values of LL and TK were calculated as previously stated by Vialle et al(10)

$\mathrm{LL}=\mathbf{0 . 6 7 P I + 2 3 . 7}$ and $\mathrm{TK}=\mathbf{0 . 1 5 P I + 4 3}$. Then the rate of occurrences of PJK to patients whose immediate postoperative $L L$ and TK values were equal or nearly equal to their theoretical value was compared to patients whose LL and TK values deviated far from their theoretical value. (discrepancy of $3^{0}$ of a real value from theoretical value was accepted). Formula 2: immediate postoperative GSA was calculated by the formula; GSA=LL+TK +PI as previously reported by Yagi et al(2). The occurrence of PJK to patients with GSA $>45^{0}$ versus GSA $<45^{0}$ were compared. Formula 3: The position of the sagittal apex of $L L$ was marked on immediate postoperative radiological films. The hypothetical apex value of LL was assumed to be at $\mathrm{L} 4$ in $\mathrm{PI}<55^{\circ}$ and $\mathrm{L} 3$ when $\mathrm{PI}>55^{\circ}$ as stated by Roussouly et al(11). Then the occurrences of PJK between patients whose sagittal apexes of LL were at theoretical value were compared to patients whose sagittal apexes of LL were not at their theoretical value.

Formula 4: The sum of LL and TK was calculated from the immediate postoperative radiological films as stated by Mendoza et al(12). The occurrences of PJK between positive-sum (LL + TK) and negative-sum $(\mathrm{LL}+\mathrm{TK})$ were compared. PJK angle was calculated from radiological films in the final follow-up visit as previously described by Glattes et al(3).

\section{Statistical analysis}

IBM SPSS (version 21) was used for statistical analysis. Fisher exact test was performed for continuous variables and the Chi-square test was used for noncontinuous variables. The odds ratio (OR) was 
calculated. A p-value of $<0.05$ was considered to be statistically significant.

\section{Results}

52 patients fulfilled our inclusion criteria, the ratio of female to male was $16.3: 1$, The mean age for all cases, was $61.1 \pm 6$.3. The mean follow-up time in years was $2.05 \pm 0.85$. The mean time in years for the occurrence of PJK was $1.25 \pm 0.61$, (0.5-3), with $71.43 \%$ of cases occurring within 1 year postoperatively. There were 14 cases of PJK, the incidence of PJK was $26.9 \%$. The mean age for PJK cases was $63.2 \pm$ 5.2 years and non-PJK cases were $60.3 \pm 6.6(p=0.14)$. The mean PI was $54.64 \pm 13.91$. The mean PT preoperative and postoperative were respectively $22.98 \pm 8.08$ and $19.28 \pm 7.64,(p=0.018)$. The average LL preoperatively and postoperatively were respectively $-41.26 \pm 10.95$ and $-54.25 \pm 11.41$, $(p \otimes 0.05)$. The average preoperative and postoperatively TK were respectively $39.76 \pm 12.17$ and $14.91 \pm 14.91(\mathrm{p}=$ 0.076). The average preoperative and postoperative SS were respectively $29.99 \pm 10.44$ and $33.84 \pm 9.73$ $(p=0.054)$. UIV was located in upper thoracic (T1-T4) in $67.3 \%$, in mid-thoracic (T5-T9) in $25 \%$, and thoracolumbar (T10 -L2) in 7.7\%. The rate of occurrences of PJK in these regions was respectively $25.71 \%, 30 \%$, and $25 \%(p=0.94)$. The preoperative LL apex was located at L2 in $1.92 \%$ patients, L2-L3 disc in $21.15 \%$ patients, L3 in $26.92 \%$ patients, L3-L4 disc in 25\% patients, and L4 in 25\% patients. The occurrences of PJK as speculated by different formulae from immediate postoperative hypothetical calculated value were as follows: Formula 1: The mean theoretical value of $L L$ as calculated from Vialle et al method was $-60.31 \pm 9.31$, different from the true value $-54.25 \pm 11.41(p=0.004)$. And the mean theoretical value of TK was $51.2 \pm 2.08$ different from the real value $44.54 \pm 14.91(P=0.002)$. PJK occurred in $26 \%$ of patients with postoperative LL and Tk equal or nearly equal to their hypothetical value. While PJK occurred in $27.6 \%$ of patients when $L L$ and TK were far different from their theoretical value. Odds ratio (OR) 1.08, 95\% confidence interval $(\mathrm{Cl}),(0.31-3.72),(P=0.9)$. Formula 2: The mean GSA was $44.93 \pm 18.6$. Among $44.23 \%$ of the patients had GSA $<45^{\circ}$ and $55.77 \%$ of the patients had $G S A>45 \%$. PJK occurred in $16 \%$ of cases with GSA $<45^{\circ}$ and $34.5 \%$ of cases with $\mathrm{GSA}>45^{\circ}$. OR $=2.5,(95 \% \mathrm{Cl}, 0.67-$ 9.38), $(P=0.17)$. Formula 3: postsurgical sagittal apexes of lumbar lordosis were located at a hypothetical position in 24 cases, and $12.5 \%$ of these cases developed PJK. While sagittal apexes were not located at the hypothetical position in 28 cases and $39.3 \%$ of the cases developed PJK. P $=0.03,0 R$ 4.53, (95\% Cl 1.09-18.9). Formula 4: The mean sum of ( $L L+T K)$ was $-9.7 \pm 17.4$. The sum (LL + TK) was positive in 14 patients and $28.6 \%$ of these patients developed PJK. and the sum (LL + TK) was negative in 38 patients, $26.3 \%$ of the patients developed PJK. OR $=1.12,95 \% \mathrm{Cl}(0.29-4.31), \mathrm{P}=0.87$.

The occurrences of PJK as predicted by each formula are shown in Fig. 1 below. 
Table 1

Preoperative and postoperative spinal pelvic parameters in degree.

\begin{tabular}{|c|c|c|c|c|c|}
\hline & & Preoperative & postop & rative & \\
\hline & MEAN & SD & MEAN & SD & P-value \\
\hline Pelvic incidence & 54.64 & 13.91 & & & \\
\hline Pelvic tilt & 22.98 & 8.08 & 19.28 & 7.64 & 0.018 \\
\hline Lumbar lordosis & -41.26 & 10.95 & -54.25 & 11.41 & $\leq 0.05$ \\
\hline Thoracic kyphosis & 39.76 & 12.17 & 44.54 & 14.91 & 0.076 \\
\hline Sacral slope & 29.99 & 10.44 & 33.84 & 9.73 & 0.054 \\
\hline
\end{tabular}

\section{Discussion}

Several risk factors for occurrences of PJK have been reported in the literature including age more than 55 years, large abnormal preoperative sagittal parameters (high lumbar lordosis and long thoracic kyphosis), application of pedicle screws, thoracoplasty procedures, big curve correction, posterior and combined anteroposterior spinal fusion (i.e., distraction of the posterior tension band and other posterior intervertebral elements), fusion involving the lower lumbar spine and sacrum, osteoporosis and high body mass index $(7,8)$. The common agreement on direct postoperative radiographic prognostic criteria for PJK has not yet been established. Maruo et al(16) suggested that improved postoperative radiographic sagittal alignment parameters are an important method to mitigate the development of PJK after adult deformity correction. In this retrospective study, we have used four formulae, to predict the development of PJK based on immediate post-operative radiological films. We found that among the four proposed formulae, the position of the sagittal apex of lumbar lordosis (formula 3 ) is the best immediate postoperative radiographic predictor for occurrences of PJK among the four hypothesized formulae. It has a great predictive value OR $4.5(95 \% \mathrm{Cl} 1.09-18.9)$. This means that the occurrence of PJK is almost 5 times high when the apex of LL is not at its theoretical position compared to when it is at its theoretical position. The second good predictor parameter in our study for the occurrence of PJK was GSA (formula 2). The predictive value for $\mathrm{GSA}$ was $\mathrm{OR}=2.5,(95 \% \mathrm{Cl}, 0.67-9.38)$. This means that when $\mathrm{GSA}$ is $>45^{0}$ the occurrence of PJK is 2.5 times compared to when GSA is $<45^{\circ}$. Even though formula 2 failed to reject the null hypothesis as the $\mathrm{Cl}$ includes 1 . The 3rd good predictor for occurrences of PJK was formula 4, when the sum positive $(L L+T K)$ was compared with negative-sum $(L L+T K)$ the predictive value was OR 1.12, (95\% $\mathrm{Cl} 0.29-4.39)$. But failed to reject the null hypothesis as the $\mathrm{Cl}$ includes 1 . The least predictive formula for occurrences of PJK among the four proposed formulae was formula 1 . When the LL and TK were equal or nearly equal to their theoretical value the predictive ability for occurrences of PJK was OR 1.08 (95\% $\mathrm{Cl} 0.31 .-3.72)$. The $\mathrm{Cl}$ includes 1 , it also failed to reject the null hypothesis. 
Lafage et al(17) in their virtual model stated that realignment surgery for the adult spinal deformity (ASD) can be extremely successful by restoring a balanced sagittal alignment. Rose et al(18) defined ideal GSA as $\mathrm{PI}+\mathrm{LL}+\mathrm{TK}$ less than $45^{\circ}$ and they found that the formula has a great sensitivity in forecasting the success of pedicle subtraction osteotomy (PSO) for two years. Yagi et al(2) in their retrospective review of 157 cases, they found that more than $80 \%$ of patient with non-ideal global sagittal alignment $\left(G S A>45^{0}\right)$ developed PJK, and the OR for these patients was 29 . Sebaaly et al(19) in their retrospective study of 250 cases of adult idiopathic scoliosis from a multicentric database, found that GSA $>45^{\circ}$ was associated with the occurrences of PJK, with OR 1.7. in this study we also found that GSA $>45^{\circ}$ is highly associated with occurrences of PJK, with OR 2.5. This might be explained by the reason that increased GSA results in the positive (SVA) and increases the stress for the proximal and distal junctions of instrumentation that lead to the development of PJK(20). Roussouly et al(11) after studying the sagittal alignment of 160 healthy volunteers divided the spine into four types based on inflexion point. Type 1 and type 2 had low $\mathrm{Pl}$, type 3 , and type 4 had high PI. They found that the lower arc of lordosis is a key determinant of global lordosis (lordosis tilt angle, location of the apex, and the number of lordotic vertebras). Both low SS and low PI are accompanied by flat and short lumbar lordosis while high SS and high PI are accompanied by long and curved lumbar lordosis. They further proposed that the spine is well balanced when lordosis apex is at L4 for low PI and L3 for high PI. In our study, the occurrences of PJK were very higher to patients whose apexes of sagittal lordosis were not positioned at theoretical position than patients whose apexes of sagittal lordosis where positioned at theoretical position. These findings are similar to what has been reported in the literature by other scholars(19). This may be explained as when the postoperative apex is located high above its theoretical position kyphosis has less room to be constructed and increase the risk of occurrences of PJK and other spinal abnormalities(21). In this study, we found that both formula 1 and formula 4 have low predictive ability for occurrences of PJK. This is similar to what has been reported recently in the literature(19).

Despite meeting the criteria of all formula above yet some patients developed PJK and, even some of the formulae failed to reject the null hypothesis. This might be explained by the reason that, the risk factors for occurrences of PJK are many. Some have been reported in the literature, including age, low bone mineral density, the use of pedicle screws, thoracoplasty, combined anteroposterior surgery, and long fusion surgery(22-24). The most important finding of this study is that the theoretical position of the sagittal apex of lumbar lordosis is a preventive factor for the development of PJK. In this study "the position of the apex of lumbar lordosis has emerged as an independent risk factor for occurrences of PJK. This finding can guide the surgeon to decide the level of pedicle subtraction osteotomy (PSO) or a suitable place to put lordotic cages during spine deformity correction. Lafage et al(25) in a multicenter retrospective study of 70 cases of adult spine deformity who underwent PSO found no difference in sagittal curve correction between L3 and L4 PSO. But in this study, we suggest that PSO be done at L4 in low PI and L3 in high PI patients.

The limitations of this study include the following: Is a retrospective study design with some data missing and some patients lost follow-up, for example, the data for bone mineral density and Oswestry 
disability index scores were not found. The study failed to compare the theoretical location of the apex of LL to other risk factors for occurrences of PJK, such as bone mineral density, body mass index, long fusion to the pelvis, etc. Also is a single-center study with a small sample size. But despite these limitations, the results from this study are similar to what has been reported by other scholars.

\section{Conclusion}

Among the four proposed formulae for predicting occurrences of PJK, the position of the sagittal apex of lumbar lordosis is an excellent predictor of the development of PJK followed by GSA. Hypothetical values of $L L$ and TK, positive or negative-sum of ( $L L+T K)$ are weak predictors for occurrences of PJK.

\section{Abbreviations}

\section{PJK}

Proximal junction kyphosis, $\mathbf{L L}=$ lumbar lordosis, $\mathbf{T K}=$ thoracic kyphosis, $\mathrm{PI}=$ pelvic incidence, $\mathbf{G S A}=$ global sagittal alignment, UIV = uppermost instrumented vertebrae, SRS = scoliosis research society, ODI = Oswestry disability index, SVA = sagittal vertical axis, AP = anteroposterior PT = pelvic tilt, SS = Sacral slope, $\mathrm{PJA}=$ Proximal junctional angle, $\mathrm{OR}=$ odds ratio, $\mathrm{Cl}=$ confidence interval, $\mathrm{PSO}=$ pedicle subtraction osteotomy

\section{Declarations}

\section{Ethical approval and consent to participate:}

The study protocol was approved by the Ethics Committee of the Xiangya first Hospital of Central South University.

\section{Consent for publication:}

"Not applicable"

\section{Availability of data and material:}

"The datasets supporting the conclusion of this article are given as Additional file 1"

\section{Competing Interest]}

The authors declare that they have no competing interests.

\section{Funding:}

No fund was received during the preparation of this work

\section{Authors contribution:}


HQZ gave the idea and designed the study, DRM, DYX, and WYX collected the data, analyzed the data, and wrote the paper. All authors read and approved the final manuscript.

\section{Acknowledgments}

The authors cordially appreciate the assistance from the working staff of the medical record department of Xiangya's first hospital for providing the data whenever needed.

\section{Authors information}

Hongqi Zhang: Is currently head of the department of spine surgery of Xiangya hospital of Central South University.

Manini Daudi Romani: Is currently a first-year Ph.D. student in the spine department of Xiangya hospital of Central South University.

YuXiangWang; Is currently a spinal surgeon, in the department of spine surgery of Xiangya hospital of Central South University.

DuYuXuan: Is currently a second-year Ph.D. student in the spine department of Xiangya hospital of Central South University.

\section{References}

1. Cho SK, Shin JI, Kim YJ. Proximal junctional kyphosis following adult spinal deformity surgery. Eur Spine J. 2014;23(12):2726-36.

2. Yagi M, Akilah KB, Boachie-Adjei O. Incidence, risk factors and classification of proximal junctional kyphosis: surgical outcomes review of adult idiopathic scoliosis. Spine (Phila Pa 1976). 2011;36(1):E60-8.

3. Glattes RC, Bridwell KH, Lenke LG, Kim YJ, Rinella A, Edwards C, 2nd. Proximal junctional kyphosis in adult spinal deformity following long instrumented posterior spinal fusion: incidence, outcomes, and risk factor analysis. Spine (Phila Pa 1976). 2005;30(14):1643-9.

4. Cammarata M, Aubin CE, Wang X, Mac-Thiong JM. Biomechanical risk factors for proximal junctional kyphosis: a detailed numerical analysis of surgical instrumentation variables. Spine (Phila Pa 1976). 2014;39(8):E500-7.

5. Lau D, Clark AJ, Scheer JK, Daubs MD, Coe JD, Paonessa KJ, et al. Proximal junctional kyphosis and failure after spinal deformity surgery: a systematic review of the literature as a background to classification development. Spine (Phila Pa 1976). 2014;39(25):2093-102.

6. Lee J, Park YS. Proximal Junctional Kyphosis: Diagnosis, Pathogenesis, and Treatment. Asian Spine J. 2016;10(3):593-600. 
7. Bridwell KH, Lenke LG, Cho SK, Pahys JM, Zebala LP, Dorward IG, et al. Proximal junctional kyphosis in primary adult deformity surgery: evaluation of 20 degrees as a critical angle. Neurosurgery. 2013;72(6):899-906.

8. O'Shaughnessy BA, Bridwell KH, Lenke LG, Cho W, Baldus C, Chang MS, et al. Does a long-fusion "T3sacrum" portend a worse outcome than a short-fusion "T10-sacrum" in primary surgery for adult scoliosis? Spine (Phila Pa 1976). 2012;37(10):884-90.

9. Hassanzadeh H, Gupta S, Jain A, El Dafrawy MH, Skolasky RL, Kebaish KM. Type of Anchor at the Proximal Fusion Level Has a Significant Effect on the Incidence of Proximal Junctional Kyphosis and Outcome in Adults After Long Posterior Spinal Fusion. Spine Deformity. 2013;1(4):299-305.

10. Vialle R, Levassor N, Rillardon L, Templier A, Skalli W, Guigui P. Radiographic analysis of the sagittal alignment and balance of the spine in asymptomatic subjects. The Journal of bone and joint surgery American volume. 2005;87(2):260-7.

11. Roussouly P, Gollogly S, Berthonnaud E, Dimnet J. Classification of the normal variation in the sagittal alignment of the human lumbar spine and pelvis in the standing position. Spine (Phila Pa 1976). 2005;30(3):346-53.

12. Mendoza-Lattes S, Ries Z, Gao Y, Weinstein SL. Proximal junctional kyphosis in adult reconstructive spine surgery results from incomplete restoration of the lumbar lordosis relative to the magnitude of the thoracic kyphosis. The lowa orthopaedic journal. 2011;31:199-206.

13. Gandevia B, Tovell A. DECLARATION OF HELSINKI. The Medical journal of Australia. 1964;2:320-1.

14. Lafage V, Ames C, Schwab F, Klineberg E, Akbarnia B, Smith J, et al. Changes in thoracic kyphosis negatively impact sagittal alignment after lumbar pedicle subtraction osteotomy: a comprehensive radiographic analysis. Spine (Phila Pa 1976). 2012;37(3):E180-7.

15. Legaye J, Duval-Beaupere G, Hecquet J, Marty C. Pelvic incidence: a fundamental pelvic parameter for three-dimensional regulation of spinal sagittal curves. Eur Spine J. 1998;7(2):99-103.

16. Maruo K, Ha Y, Inoue S, Samuel S, Okada E, Hu SS, et al. Predictive factors for proximal junctional kyphosis in long fusions to the sacrum in adult spinal deformity. Spine (Phila Pa 1976). 2013;38(23):E1469-76.

17. Lafage R, Bess S, Glassman S, Ames C, Burton D, Hart R, et al. Virtual Modeling of Postoperative Alignment After Adult Spinal Deformity Surgery Helps Predict Associations Between Compensatory Spinopelvic Alignment Changes, Overcorrection, and Proximal Junctional Kyphosis. Spine (Phila Pa 1976). 2017;42(19):E1119-e25.

18. Rose PS, Bridwell KH, Lenke LG, Cronen GA, Mulconrey DS, Buchowski JM, et al. Role of pelvic incidence, thoracic kyphosis, and patient factors on sagittal plane correction following pedicle subtraction osteotomy. Spine (Phila Pa 1976). 2009;34(8):785-91.

19. Sebaaly A, Riouallon G, Obeid I, Grobost P, Rizkallah M, Laouissat F, et al. Proximal junctional kyphosis in adult scoliosis: comparison of four radiological predictor models. Eur Spine J. 2018;27(3):613-21. 
20. Yagi M, King AB, Boachie-Adjei O. Incidence, Risk Factors, and Natural Course of Proximal Junctional Kyphosis. Spine. 2012;37(17):1479-89.

21. Yu M, Silvestre C, Mouton T, Rachkidi R, Zeng L, Roussouly P. Analysis of the cervical spine sagittal alignment in young idiopathic scoliosis: a morphological classification of 120 cases. Eur Spine J. 2013;22(11):2372-81.

22. Lowe TG, Kasten MD. An analysis of sagittal curves and balance after Cotrel-Dubousset instrumentation for kyphosis secondary to Scheuermann's disease. A review of 32 patients. Spine (Phila Pa 1976). 1994;19(15):1680-5.

23. Kim YJ, Lenke LG, Bridwell KH, Kim J, Cho SK, Cheh G, et al. Proximal junctional kyphosis in adolescent idiopathic scoliosis after 3 different types of posterior segmental spinal instrumentation and fusions: incidence and risk factor analysis of 410 cases. Spine (Phila Pa 1976). 2007;32(24):2731-8.

24. Wang J, Zhao Y, Shen B, Wang C, Li M. Risk factor analysis of proximal junctional kyphosis after posterior fusion in patients with idiopathic scoliosis. Injury. 2010;41(4):415-20.

25. Lafage V, Schwab F, Vira S, Hart R, Burton D, Smith JS, et al. Does vertebral level of pedicle subtraction osteotomy correlate with degree of spinopelvic parameter correction? J Neurosurg Spine. 2011;14(2):184-91.

\section{Figures}



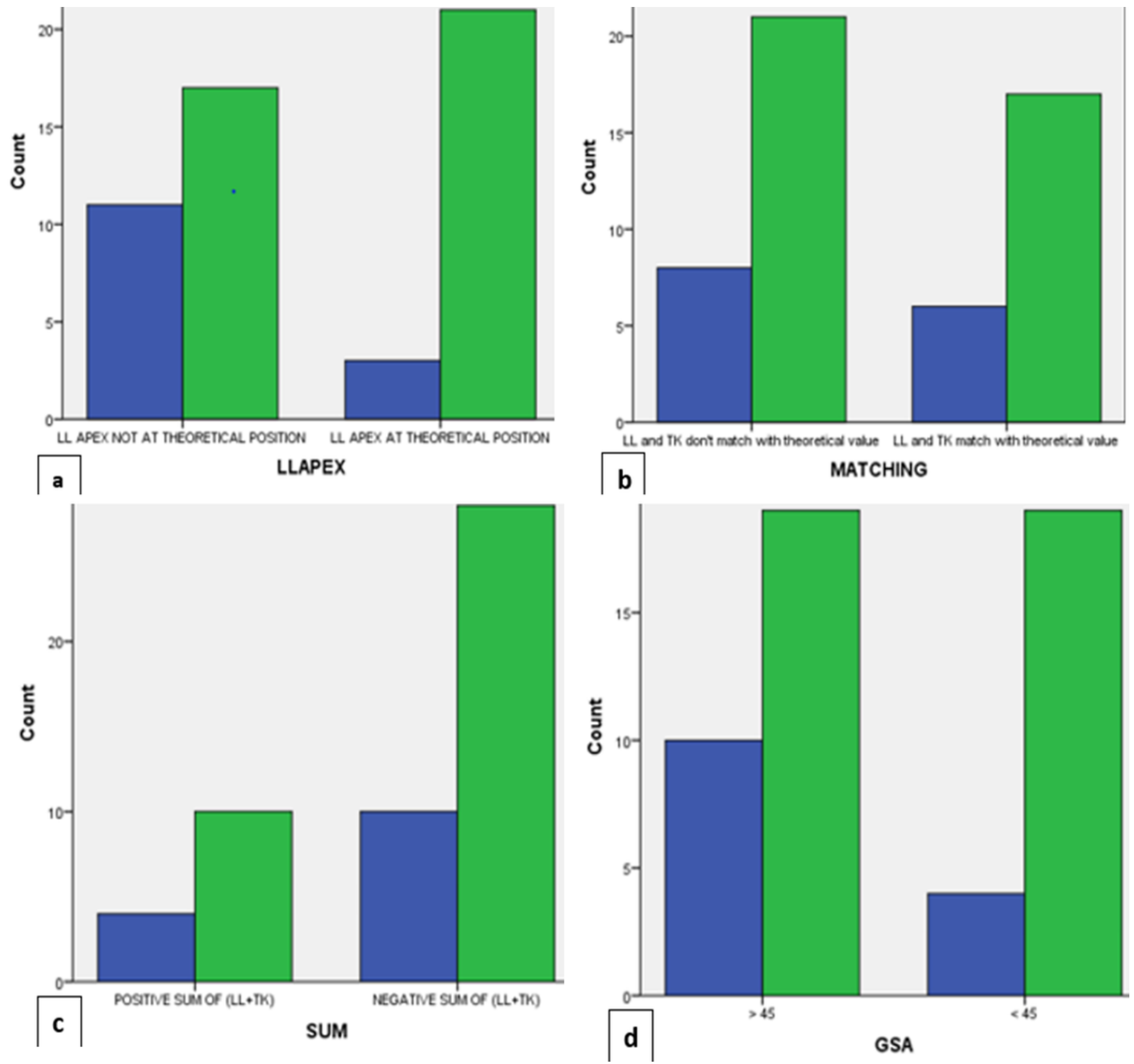

Figure 1

Compound bar charts ( $\mathrm{a} b \mathrm{c}$ and $\mathrm{d}$ ) to show the occurrences of PJK as predicted by each formula. $\mathrm{LL}=$ lumbar lordosis, TK= thoracic kyphosis, GSA = global sagittal alignment, PJK = proximal junctional kyphosis. The blue bar shows the number of patients who developed PJK while the green bar shows the number of patients who did not develop PJK 


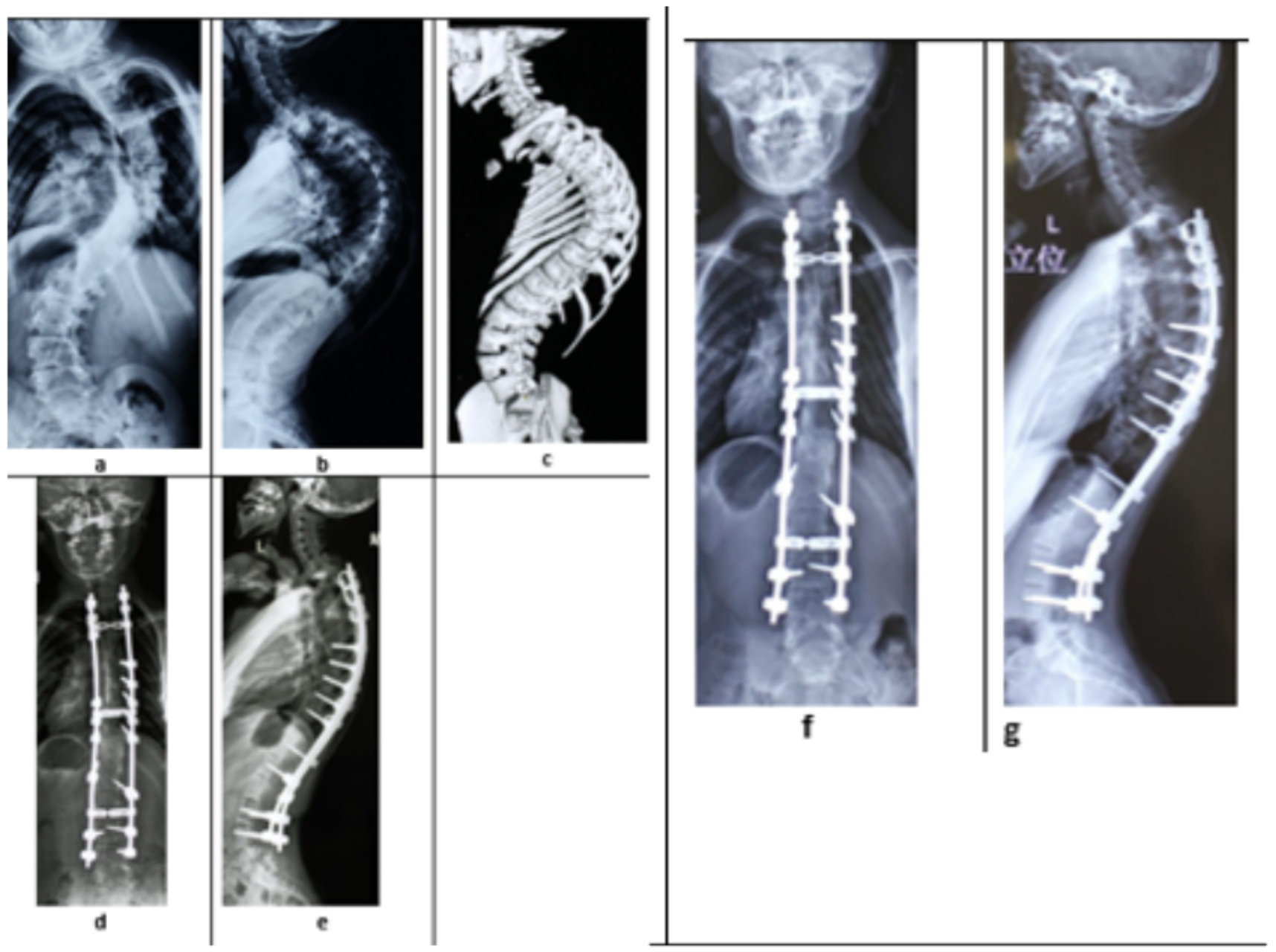

Figure 2

Radiographic films of a 40-year-old female patient with neglected scoliosis who underwent scoliosis curve correction and developed asymptomatic PJK after one year. "a" is preoperative anteroposterior (AP) $X$-ray views, " $b$ " is preoperative lateral $X$-ray view and " $c$ " is a bone scan 3D model done preoperatively. " $d$ " and " $e$ " are immediate postoperative AP and lateral $x$-ray films and " $f$ " and $g$ are AP and lateral $x$-ray films done after 1 year. The PJK angle after 1 year was 110 greater than immediate postoperative

\section{Supplementary Files}

This is a list of supplementary files associated with this preprint. Click to download.

- Additionalfile1.xlsx

- Additionalfile2.docx 